\title{
Treatment of Advanced Non-small-Cell Lung Cancer with Qi-Nourishing Essence- Replenishing Chinese Herbal Medicine Combined with Chemotherapy
}

Yabin Gong ${ }^{1,3}$, Zhenye X $\mathrm{u}^{1,4^{*}}$, Changjuan Jin ${ }^{2}$, Haibin Deng ${ }^{1}$, Zhongqi Wang ${ }^{1}$, Weidong Zhou ${ }^{1}$, Ming Zhang ${ }^{2}$, Xiaozhen Zhao ${ }^{1}$ and Lifang Wang ${ }^{1}$

\begin{abstract}
Background: To evaluate the effect of qi-nourishing essence-replenishing Chinese herbal medicine combined with chemotherapy in survival of advanced non-small-cell lung cancer(NSCLC) patients with essence and qi deficiency.

Methods: A prospective multi-centered randomized controlled study was conducted, and 266 advanced NSCLC patients were enrolled. 126 patients in control group received Vinorelbine plus cisplatin(NP) chemotherapy combined with symptom-oriented Chinese herbs medication(without qi-nourishing essence-replenishing herbs);140 patients in experimental group received NP chemotherapy combined with qi-nourishing essence-replenishing Chinese herbal medication(Kangliu Zengxiao Decoction and modified Feiyanning Decoction, during and after chemotherapy respectively).
\end{abstract}

Results: One patient in control and 2 in experimental group were excluded for failure to complete two cycles of chemotherapy. During follow-up, 17 and 7 patients in control and experimental group were excluded respectively(4 and 4 for taking Gefetinib after disease progression, 4 and 2 for receiving other chemotherapeutic regimens, 9 and 1 for lost to follow-up). 239 patients were included in the final analysis (131 in experimental group and 108 in control). Median overall survival in experimental group was significantly longer than control group (14.87vs.12.97 months, $P=0$. 027). In experimental and control group, 1-year, 3-year, 5-year, 7-year, and 9-year survival rates were 57\% vs. 53\%, 17\% vs. $8 \%, 10 \%$ vs. $2 \%, 6 \%$ vs. $0 \%$, and $6 \%$ vs. $0 \%$, respectively.

Conclusion: Qi-nourishing essence-replenishing Chinese herbal medicine combined with chemotherapy improves survival of advanced NSCLC patients with essence and qi deficiency.

Keywords: Qi-nourishing essence-replenishing, Chinese herbal medicine, Essence and qi deficiency, Chemotherapy, Non-small-cell lung cancer

\footnotetext{
* Correspondence: xuzhenye1947@126.com

1Department of Oncology, Longhua Hospital, Shanghai University of Traditional Chinese Medicine, Shanghai ,200032, China

${ }^{4}$ Department of Oncology Longhua Hospital, Shanghai University of

Traditional Chinese Medicine, 725 Southern of Wan Ping Road, Shanghai,

China

Full list of author information is available at the end of the article
} 


\section{Background}

Lung cancer is one of the malignancies with highest morbidity and mortality in China [1]. Approximately 75-85\% of lung cancers are non-small cell lung cancers (NSCLC), including squamous carcinoma, adenocarcinoma and large cell carcinoma [2]. Most patients (70\%) are diagnosed with advanced disease [3]. Although with the advent of the third-generation chemotherapeutic drugs (gemcitabine, docetaxel, and pemetrexed), the clinical efficacy remains limited. Indeed, the overall survival of advanced NSCLC patients remains low after first-line chemotherapy. Prognosis for these patients is poor with 5-year survival rates reported as less than 5\% [4]. Platinum-based doublet chemotherapy is the standard first-line treatment for advanced NSCLC when genomic testing reveals no activating epidermal growth factor receptor (EGFR) mutations, anaplastic lymphoma kinase (ALK) or ROS1 rearrangement [5], with objective response rates between $15 \sim 30 \%$ [6, 7]. Since chemotherapy have been adopted in China, Chinese herbal medicine(CHM) is widely used in combination with chemotherapy to cope with chemotherapy-related side effects as well as prolonging the survival time of patients. Our previous studies have demonstrated that qi-nourishing essencereplenishing $\mathrm{CHM}$ combined with chemotherapy can improve short-term lesion stability, quality of life and immunity in advanced NSCLC patients [8-12]. However, its effect on the long-term survival remains unknown.

According to TCM theory, qi and essence are the fundamental substance constituting human body and maintaining physical activities [13-15]. The deficiency of qi and essence leads to malfunction of the body, with clinical manifestation of fatigue and lassitude, aching lumbus and limp legs, dizziness and tinnitus, sensitivity and intolerance to cold, spontaneous perspiration and night sweats, thirst or reduced water intake, frequent nighttime urination, thready or weak pulse, red or pink tongue with some tongue coating. The pathology of lung cancer, according to TCM, is the deficiency of qi and essence and the pathogenic factors resulting from it. Therefore, the key point of TCM cancer treatment is to strengthen qi and essence and eliminate pathogenic factors, and qi-nourishing essence-replenishing CHM has been proved with satisfactory short-term effect [9].

In this study, a perspective randomized single-blinded clinical trial was conducted to evaluate the survival benefit of qi-nourishing essence-replenishing CHM combined with Vinorelbine + Cisplatin (NP) regimen in advanced NSCLC patients with essence and qi deficiency, in comparison to symptom-oriented CHM (without qi-nourishing essence-replenishing herbs) with NP regimen. The findings should help to prolong the overall survival time for advanced NSCLC patients.

\section{Methods}

\section{Patient Selection}

Source of Patients

Patients admitted in 1) Department of Oncology, Longhua Hospital, Shanghai University of Traditional Chinese Medicine; 2) Department of Integrated Traditional Chinese and Western Medicine,Shanghai Pulmonary Cancer Clinical Medicine Center, Shanghai Chest Hospital; 3) Department of Oncology, Shanghai Pulmonary Hospital, who received treatments between January 2006 and December 2012, were enrolled. The protocol was approved by Shanghai Pudong food and drug administration,All participants signed informed consent forms.

\section{Inclusion Criteria}

1. definitive diagnosis of NSCLC based on imaging, pathological, or cytological evidence;

2. diagnosis of inoperable stage IIIb-IV or postoperative recurrent metastatic NSCLC based on the Union for International Cancer Control (UICC) TNM Classification for Malignant Tumors (6th edition) [16];

3. Eastern Cooperative Oncology Group (ECOG) performance status (PS) score $\leq 2$ with expectation to complete 2-4 cycles of chemotherapy and survive more than 4 months;

4. aged between 18 and 75 years;

5. matching the traditional Chinese medicine (TCM) syndrome differentiation of essence and qi deficiency based on the TCM syndrome differentiation of primary bronchial lung cancer in the Guidelines of clinical research on Chinese new herbal medicine [17]:

The main symptoms/signs included: a) fatigue and lassitude; b) aching lumbus and limp legs; c) dizziness and tinnitus; d) sensitivity and intolerance to cold; e) red or pink tongue with some tongue coating.

Secondary symptoms/signs included: a) spontaneous perspiration and night sweats; b) thirst or reduced water intake; c) frequent night-time urination; d) thready or weak pulse.

A definitive diagnosis was made for any patient showing at least three main and one secondary symptoms/ signs, or two main and two secondary symptoms/signs.

\section{Exclusion Criteria}

1. unfulfillment of inclusion criteria;

2. severe complications of heart, liver, kidney and/or blood diseases;

3. severe infections;

4. failure to complete the 2 chemotherapeutic courses;

5. PS score $>2$; 
6. not matching the TCM syndrome differentiation of essence and qi deficiency.

\section{Case Exclusion Criteria}

1. violating the protocol criteria;

2. not matching the inclusion criteria but enrolled.

\section{Withdrawal Criteria}

1. receiving any targeted therapy or any other chemotherapeutic regimen due to disease progression within 3 months after study initiation;

2. exhibiting poor compliance that affected efficacy and safety assessments during the study process;

3. unsuitable to continue the study due to severe adverse events, complications or special physiological changes;

4. less than 3 months of Feiyanning Decoction administration after chemotherapy, or withdrawing from the study at patient's own discretion;

5. administering combined medications that potentially affect efficacy and safety assessments of the study medication;

6. withdrawing, lost to follow-up, or dying due to disease-irrelevant reasons before study completion.

\section{Criteria for Study Termination}

This study would be terminated in any of the following situations:

1) patients suffered from any severe adverse events;

2) any major deficiencies in the clinical protocol or significant deviation was determined to pose difficulties for evaluation.

\section{Study Methods}

\section{Randomization}

Patients were randomized into the experimental and control groups using complete randomization with SPSS16.0 software.

\section{Blinding}

Subjects were single-blinded to grouping.

\section{Source and Preparation of Medications}

1) Kangliu Zengxiao Decoction(Table 1) and Feiyanning Decoction(FYN decoction Table 2).

Kangliu Zengxiao Decoction(KLZX decoction) is composed of Astragalus mongholicus, Ganoderma lucidum, Coptis chinensis, Atractylodes chinensis, etc. Feiyanning Decoction(FYN decoction) contains Astragali Radix, Ganoderma lucidum, Polygonatum sibiricum, Herba
Table 1 Standard formula of Kang Liu Zeng Xiao decoction

\begin{tabular}{lll}
\hline Chinese name & Pharmaceutical name & Crude drug content (g) \\
\hline Huang Qi & Astragalus mongholicus & 30 \\
Huang Jing & Polygonatum sibiricum & 30 \\
Jiao Gu Lan & Gynostemma pentaphyllum & 15 \\
Ling Zhi & Ganoderma lucidum & 24 \\
Cang Zhu & Atractylodes chinensis & 9 \\
Nv Zhen Zi & Ligustrum lucidum & 15 \\
Huang Lian & Coptis chinensis & 6 \\
\hline
\end{tabular}

Epimedii, Paris polyphylla Smith, beehive, and dried toad skin. All herbs were provided by Longhua Hospital pharmacy, dissolved in $1000 \mathrm{ml}$ water, soaked for $30 \mathrm{~min}$, decocted to $200 \mathrm{ml}$ with gentle heat; then these herbs were dissolved in $500 \mathrm{ml}$ water and decocted to $100 \mathrm{ml}$. Two decoctions $(300 \mathrm{ml})$ were mixed, and aliquoted into 2 plastic bags $(150 \mathrm{ml}$ each), ready for use. The crude drug content was $1.0 \mathrm{~g} / \mathrm{ml}$.

2) Chemotherapeutic agents.

Vinorelbine (NVB) was provided as $10 \mathrm{mg} /$ bottle (Pierre Fabre, France). Cisplatin (DDP) was obtained as 20 mg/bottle (Qilu Pharmaceutical, China).

\section{Methods of Administration}

1) Control Group The NP regimen, comprising NVB $\left(25 \sim 30 \mathrm{mg} / \mathrm{m}^{2}\right.$, day 1 , day 8$)$ and DDP $(70 \sim 80 \mathrm{mg} /$ $\mathrm{m}^{2}$, divided into day 1-3) was used once every 3 weeks for 2-4 cycles. $150 \mathrm{ml}$ of modified Jupi Zhuru Decoction (Table 3)were used during the first treatment period(chemotherapeutic period), while

Table 2 Standard formula of Fei Yan Ning decoction

\begin{tabular}{lll}
\hline Chinese name & Pharmaceutical name & $\begin{array}{l}\text { Crude drug content } \\
(\mathrm{g})\end{array}$ \\
\hline $\begin{array}{l}\text { Sheng Huang } \\
\text { Qi }\end{array}$ & Astragalus mongholicus & 40 \\
Huang Jing & Polygonatum sibiricum & 30 \\
Shan Zhu Yu & Cornus Oycinalis & 15 \\
Ling Zhi & Ganoderma lucidum & 30 \\
Yin Yang & Epimedium davidii Franch & 30 \\
Huo & & \\
Nv Zhen Zi & Ligustrum lucidum & 15 \\
Bai Zhu & Rhizoma Atractylodis & 9 \\
& Macrocephalae & 9 \\
Feng Fang & Comb & 15 \\
Shan Ci Gu & Pseudobulbus Cremastraeseu & \\
Gan Chan Pi & Pleiones \\
Chong Lou & Toad Skin & 9 \\
\hline & Paris polyphylla Smith & 15 \\
\hline
\end{tabular}


Table 3 Standard formula of modified Jupi Zhuru decoction

\begin{tabular}{lll}
\hline Chinese name & Pharmaceutical name & $\begin{array}{l}\text { Crude drug content } \\
(\mathrm{g})\end{array}$ \\
\hline Dang Shen & Codonopsis pilosula & 15 \\
Chen Pi & Pericarpium citri reticulatae & 9 \\
Jiang Ban Xia & Pinellia ternata & 9 \\
Jiang Zhuru & Bambusa tuldoides Munro & 9 \\
Da Zao & Zizyphus jujuba & 9 \\
Gan Cao & Glycyrrhiza uralensis & 6 \\
\hline
\end{tabular}

$150 \mathrm{ml}$ of symptom-oriented decoction (Table 4) was administered during the second treatment period(14 days after the last course of chemotherapy). The symptomoriented decoction was completely different from that in the expeimental group, without qi-nourishing essencereplenishing herbs. The basic principles of the modification included: in patients with diarrhea, lentils and dioscorea were added; in thirsty ones, Glehnia littoralis, Cochinchinese Asparagus and Ophiopogon japonicus; for those with cough and bitter almond, Eriobotrya japonica and Rhizoma phragmitis were added; for those having trouble sleeping, Caulis Polygoni Multiflori and Albizia julibrissin were added; in subjects with lower back soreness, Dipsacus root, Herba Taxilli and Eucommia ulmoides were added; in those with palpitation, honey-fried licorice root and Salvia miltiorrhizae were added; in patients with poor appetite, chicken gizzard membrane, rice sprout and malt etc. were added.

2) Experiment Group The regimen, dose, usage, and course of the chemotherapy were the same as control group. $150 \mathrm{ml}$ KLZX decoction was orally administered twice daily during the first treatment period (chemotherapeutic period), and within14 days after the last course of chemotherapy. During the second treatment period(14 days after the last course of

Table 4 Standard formula of symptom-oriented decoction

\begin{tabular}{lll}
\hline Chinese name & Pharmaceutical name & $\begin{array}{l}\text { Crude drug content } \\
(\mathrm{g})\end{array}$ \\
\hline Xing Ren & Apricot kernel & 9 \\
Lu Gen & Reed rhizome & 30 \\
Pi Pa Ye & Folia eriobotryae & 9 \\
Dan Shen & Salvia miltrorrhiza & 15 \\
Bai Hua She She Cao & Herba hedyotis & 30 \\
Pu Gong Ying & Dandelion & 30 \\
Yu Xing Cao & Houttuynia cordata & 30 \\
Jin Yin Hua & Honeysuckle & 9 \\
Chen Pi & Pericarpium citri reticulatae & 9 \\
Xia Ku Cao & Prunella spike & 15 \\
Sheng Mu Li & Concha ostreae & 30 \\
\hline
\end{tabular}

chemotherapy), $150 \mathrm{ml}$ of modified FYN decoction was administered twice daily for one year or until intolerable side effect.

The basic principles of the modification as control group.

\section{Outcome Measure}

The main outcome measure in this study was overall survival.

\section{Statistical Analysis}

The SPSS16.0 software was used for statistical analysis. Quantitative data were expressed as mean $\pm S D$, and compared by $t$ test; qualitative data were expressed as rate, and analyzed by the $x^{2}$ or Mann-Whitney test. Kaplan-Meier curves were generated to assess survival, with the Log-rank test adopted for comparisons.

\section{Results}

\section{General Information}

The 266 subjects matching the inclusion criteria were enrolled and treated between January 2006 and December 2012. The last follow-up was December 2015, and overall follow-up rate was $90.2 \%$. One and two patients respectively in the control and experimental groups were excluded for failing to complete the two chemotherapeutic courses. Another 17 patients were further excluded in control group during follow-up, including 4, 4, and 9 patients for taking Gefetinib due to disease progression, administering other chemotherapeutic regimens, and lost to follow-up, respectively; in experimental group, 7 patients were further excluded, including 4, 2, and 1 patients, respectively, for taking Gefetinib due to disease progression, administered other chemotherapeutic regimens, and lost to follow-up. Therefore, 239 were included in the final analysis, with 131 and 108 in the treatment and control groups respectively. No significant difference was found in baseline characteristics (gender, age, staging and histology) between the two groups (Table 5).

\section{Survival Analysis}

The median survival time (MST) was 14.87 (95\% CI 11.729-18.011) and 12.97 (95\% CI 11.252-14.688) months in the experiment and control groups, respectively. ( $P=0.027$ ) (Table. 6 and Fig. 1);1-, 3-, 5-, 7-, and 9 -year survival rates were $57 \%, 17 \%, 10 \%, 6 \%, 6 \%$ in the experiment. However, $53 \%, 8 \%, 2 \%, 0 \%, 0 \%$ in control group.1-year, 3-year, 5-year, 7-year, and 9-year survival rates were improved in experiment group(Table. 7). 
Table 5 baseline characteristics. No significant difference was found in baseline characteristics (gender, age, staging and histology) between the two groups

\begin{tabular}{llll}
\hline Characteristic & $\begin{array}{l}\text { Treatment group } \\
(n=131)\end{array}$ & $\begin{array}{l}\text { Control group } \\
(n=108)\end{array}$ & $P$ value \\
\hline Sex-n(\%) & & $65(61.11)$ & \\
$\quad$ Male & $80(61.06)$ & $42(38.89)$ & \\
Female & $51(38.94)$ & & 0.889 \\
Age & & $60.56(41-80)$ & \\
Median(years) & $60.89(40-78)$ & $75(56)$ & \\
$<65$ (\%) & $89(54.3)$ & $33(44)$ & \\
$\geq 65$ (\%) & $42(45.7)$ & & 0.367 \\
Disease stages at entry-n(\%) & & $4(3.7)$ & \\
IIlla & $2(1.5)$ & $34(31.5)$ & \\
IIIb & $35(26.7)$ & $70(64.8)$ & \\
IV & $94(71.8)$ & & \\
Histology-n(\%) & & $74(68.5)$ & \\
Adenocarcinoma & $87(66.4)$ & $22(20.4)$ & \\
Squamous carcinoma & $29(22.1)$ & $0(0)$ & \\
Adenosquamous & $1(0.8)$ & $12(11.1)$ & \\
carcinoma & & & \\
Large cell carcinoma & $14(10.7)$ & & \\
and other & & & \\
\hline
\end{tabular}

\section{Median Survival}

\section{1-, 3-, 5-, 7-, and 9-Year Survival Rates}

\section{Comparison of Survival Rates}

\section{Discussion}

Lung cancer is the malignancy with highest morbidity and mortality worldwide [18], and it is urgent to improve survival in patients with advanced lung cancer. The Vinorelbine + cisplatin (NP) regimen adopted in this study is relatively low-cost, and serves as one of the first-line standardized regimens for advanced NSCLC patients without driving gene mutations such as EGFR and $A L K$ [19]. But its effect is far from satisfactory, and

Table 6 Comparison of median survival between the two groups. Log-rank test was used to compare survival between the two groups. If patients receive qi-nourishing essencereplenishing TCM after chemotherapy for more than three months and switch to Gefetinib due to disease progression, survival was calculated as time to the first day of Gefetinib administration plus one month

\begin{tabular}{llll}
\hline Group & $\begin{array}{l}\text { Median survival } \\
\text { (month) }\end{array}$ & $\begin{array}{l}\text { 95\% confident interval } \\
\text { (month) }\end{array}$ & $P$ value \\
\hline Control & $12.970 \pm 0.877$ & $11.252-14.688$ & 0.027 \\
Experiment & $14.870 \pm 1.602$ & $11.729-18.011$ & \\
\hline
\end{tabular}

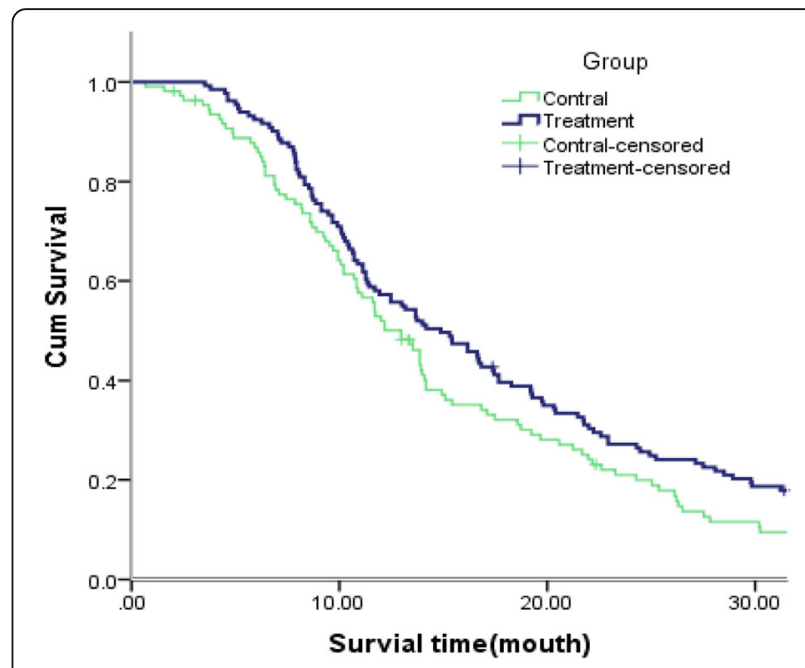

Fig. 1 Kaplan-Meier curves for survival and cumulative survival assessment. Control and experimental groups were assessed. Control-censored data, treatment-censored data; length of survival (months)

combination therapy with other agent to improve survival is in urgent need.

It is recorded in Naniing, a classical book of TCM written more than two thousand years ago, that 'treating physical weakness by warming therapy for nourishing qi and treating essence deficiency by supplying tasty and nutritional animal and plant food', 'for those with lung damages nourishing their qi', and 'for those with renal damage nourishing their kidneys'. Professor Zhenye Xu proposed the qi-nourishing essence-replenishing antitumor therapy for advanced lung cancer. Emphasizing qi-nourishing essence-replenishing methods that tonify kidney in the treatment of advanced lung cancer is based on the following TCM theory. Kidney essence is the congenital foundation and root of functional movement of human body, and the relationship between the kidney and lung is mutual generation. Spleen spirit is the postnatal foundation, and supplementing spleen nourishes lung. Therefore, nourishing the lung, spleen and kidney, so as to nourish qi and replenish essence can correct systemic deficiency. The qi-nourishing essence-replenishing method is a therapeutic technique based on the epidemiological characteristics of lung cancer, qi-blood-fluid theory, and

Table 7 Comparison of 1-, 3-, 5-, 7-, and 9-year survival rates between the two groups. 1-year, 3-year, 5-year, 7-year, and 9-year survival rates were improved in experiment group

\begin{tabular}{llllll}
\hline Group (\%) & 1-year (\%) & 3-year (\%) & 5-year (\%) & 7-year (\%) & 9-year (\%) \\
\hline Control & 53 & 8 & 2 & 0 & 0 \\
Experiment & 57 & 17 & 10 & 6 & 6 \\
\hline
\end{tabular}


visceral manifestation theory, and improves clinical outcome of lung cancer.

KLZX decoction is a TCM formula developed by Prof. Zhenye $\mathrm{Xu}$ based on the therapeutic rules of replenishing qi and replenishing essence in combination with activating the spleen to clear heat and dissipate dampness. KLZX decoction can reduce toxicity and enhance efficacy during the chemotherapeutic period. It is mainly composed of Astragali Radix, Polygonatum sibiricum, Ganoderma lucidum, Coptis chinensis Franch, and Atractylodes lancea. Astragali Radix is sweet, mildly warm, and important herb for replenishing qi. Polygonatum sibiricum is a sweet and neutral herb that tonifies deficiency, neutralizes heat or cold, and replenishes essence and marrow, and therefore invigorates the kidney, replenishes essence, and nourishes the lung and spleen. Ganoderma lucidum nourishes and strengthens the body, benefits blood and qi, and replenishes essence and marrow. Atractylodes lancea is an important herb for invigorating the spleen and eliminating dampness. Coptis chinensis can clear heat and dampness in the middle energizer, exerting an effect of invigorating the spleen to eliminate dampness and regulating the stomach.

FYN decoction [8], a Fuzheng and pathogen-eliminating decoction used after the chemotherapeutic period, is expected to treat both the symptoms and root causes, prolonging cancer patient survival via its components of qi-nourishing essence-replenishing kidney-tonifying Chinese herbs and antitumor detoxifying pathogen-eliminating Chinese herbs.

Our previous studies [8-12] have demonstrated that therapy of qi-nourishing essence-replenishing CHM by stages combined with chemotherapy can improve short-term lesion stability, quality of life and immunity in advanced NSCLC patients. The present study demonstrated that it also prolongs overall survival and improves the 1-, 3-, 5-, 7-, and 9-year survival rates.

Our study showed that the efficacy of CHM therapy as modified KLZX decoction in the first treatment period for toxicity reducing and efficacy enhancing [11], while modified FYN decoction in the second treatment period as a maintenance regimen is noninferior to pemetrexed, without adverse events such as nausea, vomiting, and bone marrow suppression [20, 21]. In addition, it has lower costs compared with pemetrexed. The 5-year survival rate observed in this study (10\%) was significantly higher than that reported in other studies (2\%), and a 9-year survival rate of $6 \%$ was obtained. However, evidences from controlled randomized clinical trials are required to compare modified FYN decoction and pemetrexed for maintenance chemotherapy.

\section{Conclusion}

3.1 Qi-nourishing essence-replenishing $\mathrm{CHM}$ combined with NP chemotherapy prolongs median survival in advanced NSCLC patients with essence and qi deficiency.

3.2 Qi-nourishing essence-replenishing CHM combined with NP chemotherapy improves the 1-, 3-, 5-, 7-, and 9-year survival rates of advanced NSCLC patients with essence and qi deficiency.

\section{Abbreviations}

ALK: Anaplastic Lymphoma Kinase; NP: Vinorelbine + Cisplatin „; CHM: Chinese Herbal Medicine; NSCLC: Non-Small-Cell Lung Cancer;" ECOG: Eastern Cooperative Oncology Group;; EGFR: Epidermal Growth Factor Receptor;; KLZX: Kangliu Zengxiao Decoction;FYN:Feiyanning Decoction;"

NVB: Vinorelbine; DDP:Cisplatin.; PS: Performance Status;TCM:Traditional Chinese Medicine;

\section{Acknowledgements}

Lu Yan, Jiao Lijing helped with this manuscript modification, Chen Peiqi helped with Statistical analysis.

\section{Funding}

Funded by Shanghai Science and Technology Commission of Science and Technology Project (02-41119024); Shanghai Economic Commission New Drug Development Project(02ZDJ002).

\section{Availability of data and materials}

All data in this paper is from our present study and publications found on CNKI, Wanfang Data and pubmed.

\section{Authors' contributions}

$X X Z Y$ and JCJ chose this subject and made this research project. DHB, WZQ, $Z W D$, ZM selected and treated the patients according to the study scheme, $G Y B, Z X Z, Z M$ and WLF treated the patients and wrote the manuscript. GYB, $\mathrm{DHB}, \mathrm{ZM}$ helped with patients follow up, and provided valuable opinions on the writing of the manuscript. All authors read and approved the final manuscript.

\section{Ethical approval and consent to participate}

We did not apply for the ethics approval from Long Hua Hospital ethics committee because the study design obeyed the rule of 'Ethics, consent and permissions', and approved by the Food and Drug Administration of Shanghai City. Consent to publish were obtained from all the participants.

Consent for publication

All authors read and approved the final manuscript for publication.

\section{Competing interests}

The authors declare that they have no competing interests.

\section{Publisher's Note}

Springer Nature remains neutral with regard to jurisdictional claims in published maps and institutional affiliations.

\section{Author details}

'Department of Oncology, Longhua Hospital, Shanghai University of Traditional Chinese Medicine, Shanghai, 200032, China. 'Department of Integrated Chinese and Western Medicine, Shanghai Chest Hospital, Shanghai ,200030, China. ${ }^{3}$ Department of Oncology, Yueyang Hospital of Integrated Traditional Chinese and Western Medicine, Shanghai University of Traditional Chinese Medicine, Shanghai ,200437, China. ${ }^{4}$ Department of Oncology Longhua Hospital, Shanghai University of Traditional Chinese Medicine, 725 Southern of Wan Ping Road, Shanghai, China. 
Received: 8 July 2017 Accepted: 12 October 2017

\section{Published online: 01 April 2018}

\section{References}

1. Chen W, Zheng R, Baade PD, et al. Cancer statistics in China,2015. CA Cancer J Clin. 2016;66:115-32

2. Herbst RS, Heymach JV, Lippman SM. Lung cancer. N Engl J Med. 2008;359: 1367-80.

3. Howlader $\mathrm{N}$, Noone AM, Krapcho M, et al. SEER cancer statistics review, 1975-2012. National Cancer Institute; 2015.

4. Gulley JL, Spigel D, Kelly K, et al. Avelumab (MSB0010718C), an anti-PD-L1 antibody, in advanced NSCLC patients: a phase 1b, open-label expansion trial in patients progressing after platinum-based chemotherapy. J Clin Oncol. 2015;33:suppl; abstr 8034.

5. Aisner DL, Marshall CB. Molecular pathology of non-small cell lung cancer: a practical guide. Am J Clin Pathol. 2012;138:332-46.

6. Schiller $\mathrm{JH}$, Harrington $\mathrm{D}$, Belani $\mathrm{CP}$, et al. Comparison of four chemotherapy regimens for advanced non-small-cell lung cancer. $\mathrm{N}$ Engl J Med. 2002;346:92-8.

7. Kelly K, Crowley J, Bunn PA Jr, et al. Randomized phase III trial of paclitaxel plus carboplatin versus vinorelbine plus cisplatin in the treatment of patients with advanced non-small-cell lung cancer: a southwest oncology group trial. J Clin Oncol. 2001;19:3210-8.

8. $\mathrm{Xu} Z$, Jin C, Shen D, et al. Clinical study on treatment of advanced nonsmall-cell lung cancer with Chinese herbal medicine in different stages combined with chemotherapy [J]. Chinese Journal of Integrated Traditional and Western Medicine. 2007;27(10):874-8.

9. Xu Z. Exploration in the treatment of advanced non-small-cell lung cancer with qi-nourishing essence-replenishing Chinese herbal medicine by stages [J]. Journal of Shanghai University of traditional. Chin Med. 2010;24(4):1-4.

10. Wu J, Xu Z, Wang Z,et al.Clinical study on treatment of advanced non-small cell lung cancer with Kangliu Zengxiao decoction [J]. Chinese Journal of Integrated Traditional and Western Medicine. 2010;30(02):137-40.

11. $X u Z Y$, Jin CJ, Zhou CC, et al. Treatment of advanced non-small-cell lung cancer with Chinese herbal medicine by stages combined with chemotherapy. J Cancer Res Clin Oncol. 2011;137(7):1117-22.

12. Deng $H$, Wang $Z$, Zha $X$, et al. Clinical study on treatment of advanced nonsmall cell lung cancer with "Feiyanning granule": a randomized placebocontrolled trial. Shanghai J Tradit Chi Med. 2016;(6):40-3.

13. Zhang DB, Sun LJ, Li CJ. Function and meaning of qi and essence theoretical foundation of Inner Canon of Yellow Emperor. Chin Arch Tradit Chin Med. 2006;24(5):784-6.

14. Sun GR. The logical structure of qi and essence theory in traditional Chinese medicine. Chin Arch Tradit Chin Med. 2006;24(6):981-4.

15. Lin F, Guo LL, Wang J. Expounding the functions of qi in TCM based on the effect mitochondria. Chinese Journal of Integrative Medicine. 2014;34:903-6.

16. Sobin $\mathrm{LH}$, Wittekind $\mathrm{CH}$. International Union against Cancer(UICC), TNM classification malignant Tumours. 6th ed. New York: Wiley-liss; 2002. p. 99-103.

17. Zheng $X$. etcGuidelines of clinical research on Chinese new herbal medicine. Beijing: China Medicine Science and Technology Press; 2002. p. 219.

18. Rebecca L. Siegel MPH1, Kimberly D. Cancer statistics,2017. CA Cancer J Clin. 2017;67(1):7-30.

19. Masters GA, Temin S, Azzoli CG, et al. Systemic therapy for stage IV nonsmall-cell lung cancer: American Society of Clinical Oncology clinical practice guideline update. J Clin Oncol. 2015;33(30):3488-515.

20. Gridelli C, de Marinis F, Pujol JL, et al. Safety, resource use, and quality of life in paramount: phase III study of maintenance pemetrexed versusplacebo after induction pemetrexed plus cisplatin for advanced nonsquamous nonsmall-cell lung cancer. J Thorac Oncol. 2012;7(11):1713-21.

21. Paz-Ares LG, de Marinis F, Dediu M, et al. PARAMOUNT: Final overall survival results of the phase III study of maintenance pemetrexedversus placebo immediately after induction treatment with pemetrexed plus cisplatin for advanced nonsquamous non-small-cell lung cancer. J Clin Oncol. 2013; 31(23):2895-902.

\section{Submit your next manuscript to BioMed Central and we will help you at every step:}

- We accept pre-submission inquiries

- Our selector tool helps you to find the most relevant journal

- We provide round the clock customer support

- Convenient online submission

- Thorough peer review

- Inclusion in PubMed and all major indexing services

- Maximum visibility for your research

Submit your manuscript at www.biomedcentral.com/submit 